\title{
Corrigendum to "Strategy for the Management of Diabetic Macular Edema: The European Vitreo-Retinal Society Macular Edema Study"
}

\author{
Ron A. Adelman, ${ }^{1}$ Aaron J. Parnes, ${ }^{2}$ Zofia Michalewska, ${ }^{3}$ Barbara Parolini, ${ }^{4}$ \\ Claude Boscher, ${ }^{5}$ Didier Ducournau, ${ }^{6}$ and EVRS Macular Edema Study Group ${ }^{6}$ \\ ${ }^{1}$ Yale University School of Medicine, New Haven, CT, USA \\ ${ }^{2}$ Eyecare Medical Group, Portland, ME, USA \\ ${ }^{3}$ Ophthalmic Clinic "Jasne Blonia", Lodz, Poland \\ ${ }^{4}$ Department of Ophthalmology, S. Anna Hospital, Brescia, Italy \\ ${ }^{5}$ American Hospital of Paris, Paris, France \\ ${ }^{6}$ EVRS, Nantes, France \\ Correspondence should be addressed to Ron A. Adelman; ron.adelman@yale.edu
}

Received 4 October 2015; Accepted 16 November 2015

Copyright (C) 2015 Ron A. Adelman et al. This is an open access article distributed under the Creative Commons Attribution License, which permits unrestricted use, distribution, and reproduction in any medium, provided the original work is properly cited.

Through this corrigendum, the authors declare that the EVRS Macular Edema Study Group is a coauthor of the paper entitled "Strategy for the Management of Diabetic Macular Edema: The European Vitreo-Retinal Society Macular Edema Study" [1]. The names of "Ron Adelman" and "Aaron Parnes" are corrected.

\section{Acknowledgments}

The authors of the EVRS Macular Edema Study Group are as follows: Walid Abdelghafar, Pierre Albinet, Saad Alkhalifa, Jean-Paul Amar, Oscar Aragon Harrison, Luis Arrevola, Francisco J. Ascaso, Samer Bashir, Frank Becquet, JeanPaul Berrod, Miguel Bilhoto, Silvia Bopp, Claude Boscher, Alexandra Brix, Perfecto E.O.R. Cagampang III, Slawomir Cisiecki, Jay F. Culotta, Marcel Dominguez, Giuseppe Donvito, Didier Ducournau, Ahmed El Khashab, Mohamed Nagy Elmohamady Saad, Maha Elshafei, Mario Facino, Natalia Ferreira, Ivan Fiser, Martin Flores-Aguilar, Eric Fourmaux, Evzen Fric, Wilko Friedrichs, Rino Frisina, Piotr Fryczkowski, Stratos Gotzaridis, Hülya Güngel, Frédéric Hamon, Fabian Höhn, Doan Hong Dung, Martin Hovorka, Angela Huiskamp, Mariano Iros, Slawomir Janiec, Young-Joon Jo, Muhammad
Shabbir Khan, Robert King, Dobromila Klimczak, Christine Kusserow, Guy L'Helgoual'ch, Pierre-Olivier Lafontaine, JeanFrançois Le Rouic, Jonghyun Lee, Oxana Levkina, JeanLoup Leynaud, Aliki Liaska, Selina Lin, Luis Miguel Lopez Montero, Klaus Lucke, Angelina Meireles, Maurizio Mete, Zofia Michalewska, Janusz Michalewski, Jerzy Nawrocki, Agnieszka Nowosielska, Dominik Odrobina, Khor Sirwan Omer, Özen Ayranci Osmanbasoglu, Ihab Saad Othman, Sengul Ozdek, Radu Pacurariu, Stefanie Pape, Barbara Parolini, Iwona Partyka, Enrico Peiretti, Isabel Pinxten, Prasan Rao, Armando Razzari, Daniel Roth, Armando Sandoval, Andreas Schüler, Stephen Sinclair, Peter Stalmans, Horia Stanca, Maria Stefaniotou, Yutthana Sukonthasap, Ivan Tanev, Susana Teixeira, Dimitrios Tsouris, and Zarifa Zeynalova.

\section{References}

[1] R. Adelman, A. Parnes, Z. Michalewska, B. Parolini, C. Boscher, and D. Ducournau, "Strategy for the management of diabetic macular edema: the European vitreo-retinal society macular edema study," BioMed Research International, vol. 2015, Article ID 352487, 9 pages, 2015. 


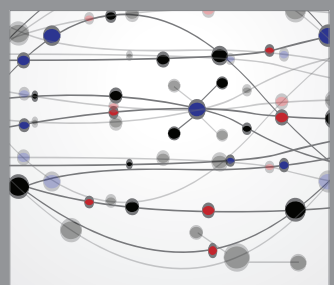

The Scientific World Journal
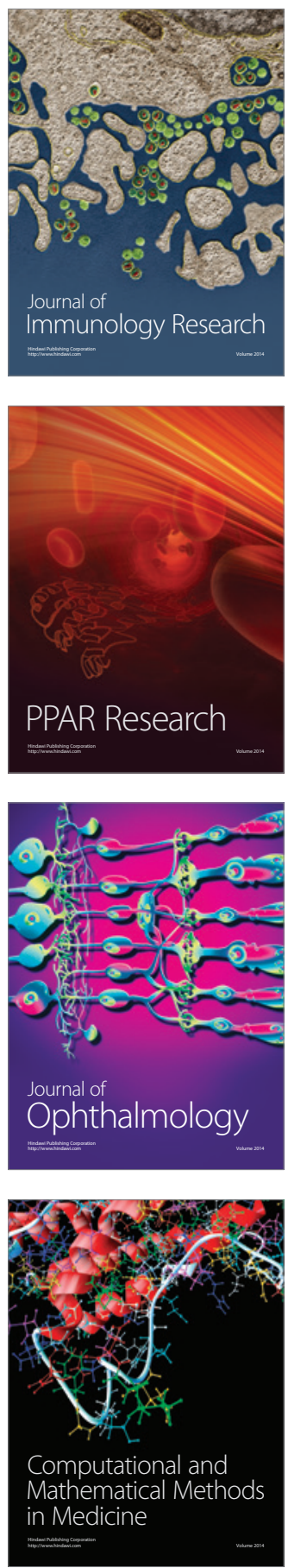

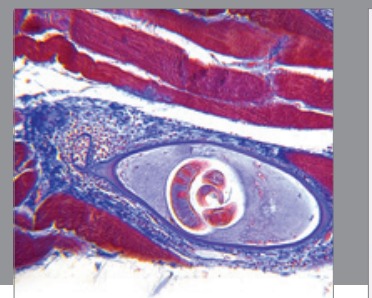

Gastroenterology

Research and Practice
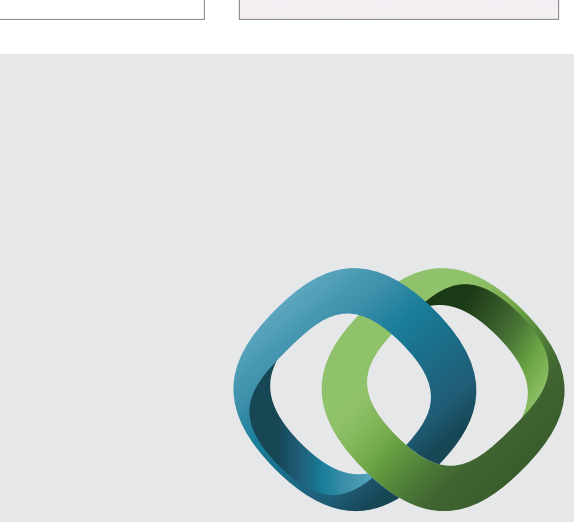

\section{Hindawi}

Submit your manuscripts at

http://www.hindawi.com
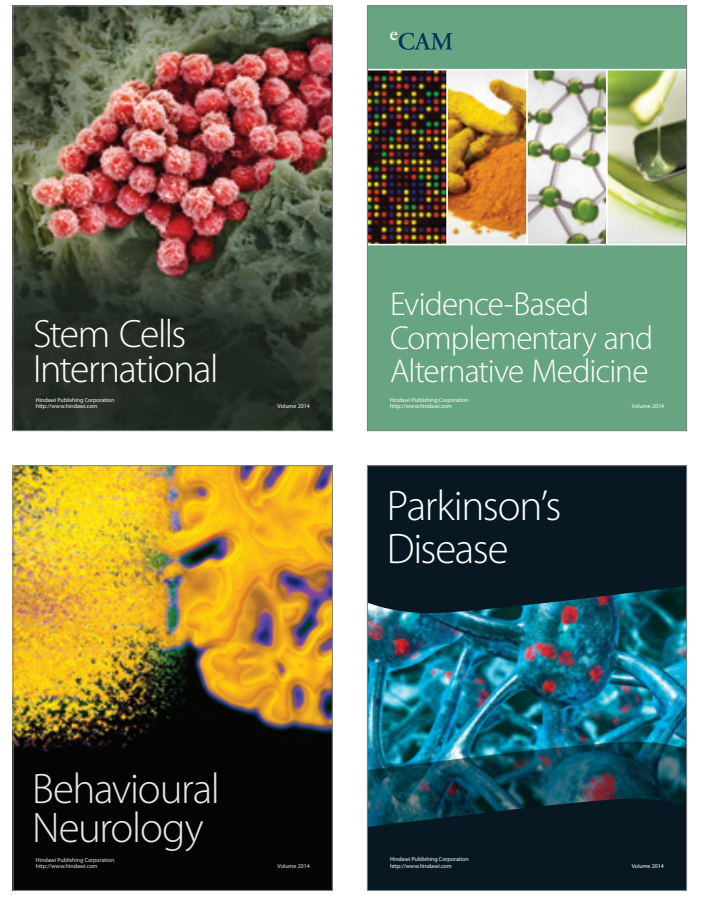
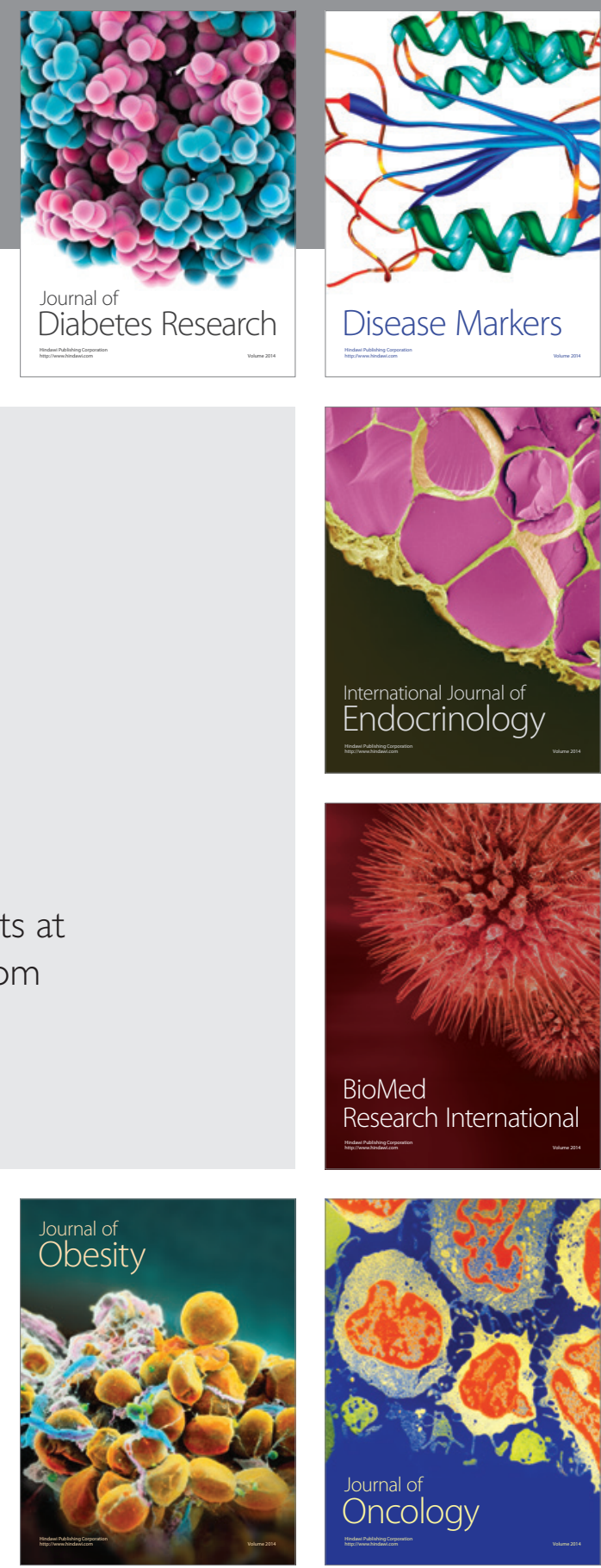

Disease Markers
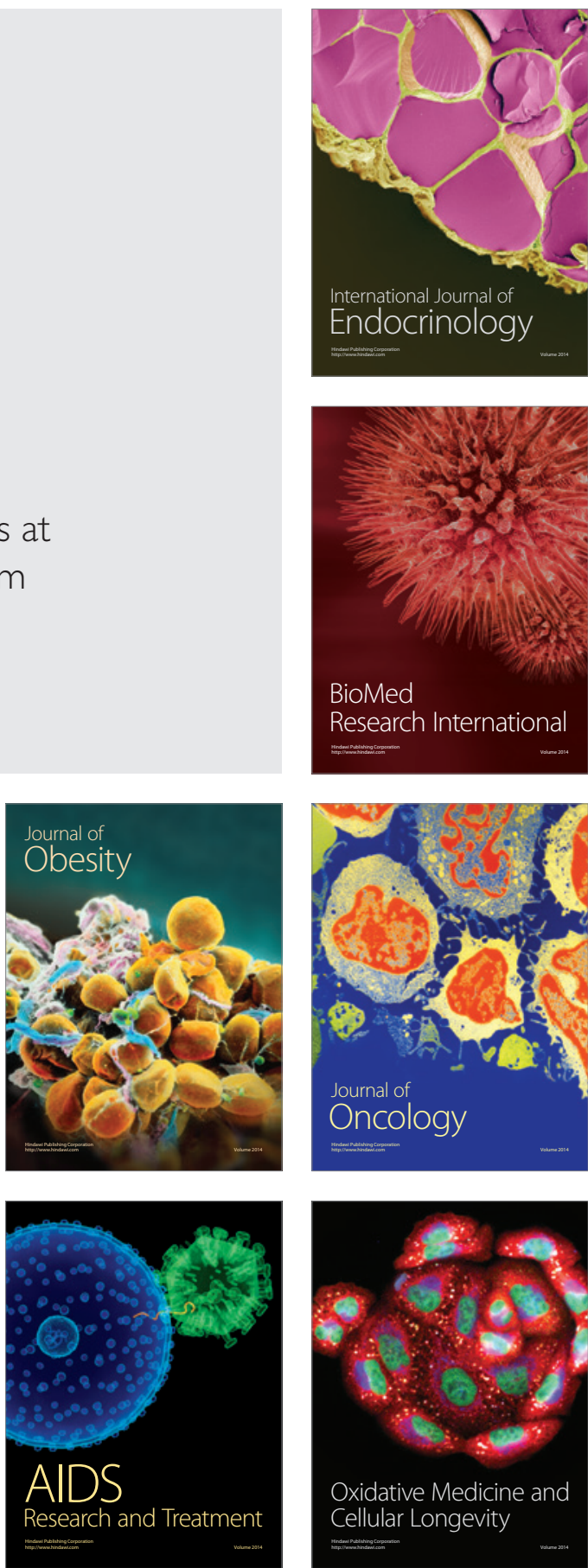